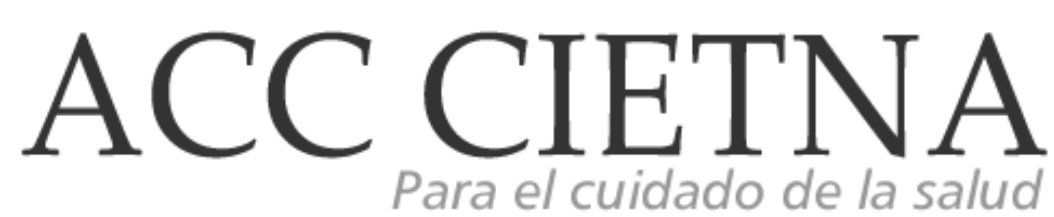

https:// doi.org/10.35383/cietna.v8i1 1.572

\title{
Nivel de adquisición de habilidades para el cuidado en internos hospitalarios de enfermería, Lambayeque 2020
}

\author{
Alarcón Vigil Gabriela Belén', Lázaro Alcántara Elaine²
}

INFORMACIÓN DEL

\section{ARTÍ́CULO}

Historia del artículo:

Recibido el 07 de abril de 2021

Aceptado el 07 de julio de 2021

\section{Palabras clave:}

Adquisición de habilidades

Internado de enfermería

Cuidado de enfermería

Habilidad (F02.784.629.131)

Cuidado (F01.752.355)

Enfermería (H02.478)

\section{RESUMEN}

Objetivo: Determinar el nivel de adquisición de habilidades para el cuidado en internos hospitalarios de enfermería, Lambayeque 2020. Método: Enfoque cuantitativo, descriptivo simple. Población constituida por 132 internos de enfermería. Se aplicó como instrumento una escala Likert, que evaluó el nivel de adquisición de habilidades para el cuidado, constituido por dos dimensiones con 21 ítems, que fue sometido a pruebas de validez, consistencia y confiabilidad, con el análisis juicio de expertos mediante la prueba binomial, llegándose a la conclusión que la proporción de preguntas aceptadas por el juez $\mathrm{N}^{\circ} 1$, 2,3 fue de 85\%, en la prueba piloto, obtuvo un alfa de Cronbach de 0.672 . Se tomó como base los criterios de rigor científico (validez interna y externa) y de los principios éticos (autenticidad, autonomía, no maleficencia y justicia). Los datos fueron procesados con el programa SPSS 25. Resultados principales: El 75,8\% aseveró que su adquisición de habilidades fue muy buena durante su internado hospitalario y el $0,8 \%$ piensa que no. En las dimensiones principiante y principiante avanzado se obtuvo un nivel de adquisición de habilidades muy buena con un $64,4 \%$ y $81,8 \%$ respectivamente. Conclusión general: la adquisición de habilidades fue muy bueno debido que los estudiantes reconocieron a sus prácticas preprofesionales como una oportunidad para lograr las competencias con la práctica constante en los diferentes servicios.

1Bachiller de enfermería de la Universidad Católica Santo Toribio de Mogrovejo, Chiclayo, Perú. Email: alarconvigilgabrielabelen@gmail.com. ORCID: https://orcid.org/0000-0003-1596-9925

2Doctora en Ciencias de Enfermería. Docente de la Universidad Católica Santo Toribio de Mogrovejo, Chiclayo, Perú. Email: elazaro@usat.edu.pe. ORCID https://orcid.org/0000-0002-7705-626X 
Level of acquisition of skills for care in nursing hospital inmates, Lambayeque 2020

ABSTRACT

Keywords:

Skills Acquisition

Nursing Internship

Nursing Care

Skill (F02.784.629.131)

Care (F01.752.355)

Nursing (H02.478)
Objective: To determine the level of acquisition of skills for care in nursing hospital interns, Lambayeque 2020. Method: Simple descriptive, quantitative approach. Population made up of 132 nursing interns. A Likert scale was applied as an instrument, which evaluated the level of acquisition of skills for care, consisting of two dimensions with 21 items, which was subjected to tests of validity, consistency and reliability, with the analysis of expert judgment through the test binomial, reaching the conclusion that the proportion of questions accepted by judge No. 1, 2.3 was $85 \%$, in the pilot test, it obtained a Cronbach's alpha of 0.672 . The criteria of scientific rigor (internal and external validity) and ethical principles (authenticity, autonomy, nonmaleficence and justice) were taken as a basis. The data were processed with the SPSS 25 program. Main results: $75.8 \%$ stated that their acquisition of skills was very good during their hospital stay and $0.8 \%$ think that it was not. In the beginner and advanced beginner dimensions, a very good level of skill acquisition was obtained with $64.4 \%$ and $81.8 \%$ respectively. General conclusion: The acquisition of skills was very good because the students recognized their preprofessional practices as an opportunity to achieve competencies with constant practice in the different services.

\section{Introducción}

La enfermería está basada en acciones, aptitudes, creatividad y razón. La esencia de enfermería es el cuidado humanizado hacia los pacientes, para ello se requiere de profesionales competentes, que realicen la labor de manera eficiente. Las habilidades y conocimientos de la enfermera se van adquiriendo con el pasar del tiempo, convirtiéndose en indispensable, para alcanzar la experticia?.

El internado, es una práctica que data de los tiempos de Florence Nightingale, quien creó el programa de formación para enfermeras en la escuela "Nightingale Training School For Nurses" afiliada al St Thomas's Hospital, su finalidad fue formar enfermeras prácticas, para brindar cuidados a enfermos pobres. Florence consideró que las alumnas de enfermería deberían realizar sus prácticas en un ambiente hospitalario, en un tiempo prolongado, el cual lo denominó "internado", estableciendo un programa de formación, donde los estudiantes debían residir en los nosocomios durante 3 años, esto con la finalidad de que se logren las competencias requeridas ${ }^{2}$.

El programa de internado en enfermería aún sigue vigente en los diferentes centros de formación universitaria del mundo, convirtiéndose en una de las oportunidades más enriquecedoras para la adquisición de experiencia preprofesional. En esta etapa se inicia la obtención de habilidades, del "novato" involucrándolo en la práctica clínica3. El interno de enfermería o "novato", es considerado como aquel que aún no cuenta con la experiencia de las diferentes situaciones que se presentan en el ámbito hospitalario, limitando el desempeño de sus tareas, sin embargo, esto no lo exime de enfrentarse a sus obligaciones, ya que sus 
conocimientos sobre el cuidado al paciente son básicos ${ }^{4}$.

En Perú, los programas de internado de enfermería se dan en el 5 to año de estudios, inicia desde la postulación de una plaza ofertada por la Gerencia Regional de Salud (GERESA), en la cual, se rinde examen de conocimientos y según los resultados se accede a elegir el Hospital, el cual les proporcionará oportunidades para la adquisición de habilidades. Los horarios están acordes con la legislación nacional, con 25 turnos de 6 a 12 horas continuas, durante 4 a 12 meses, bajo la supervisión de la institución a través de una instructora que pertenece al hospital sede.

El internado, se basa en un proceso de adquisición de habilidades, caracterizado por circunstancias que constituyen desafíos constantes para los internos, considerando que sus dominios aún podrían ser percibidos como deficientes en la toma de decisiones y débil intuición, sin embargo, con el tiempo de permanencia y la repetición de rutinas, van logrando la seguridad y capacidad para el desarrollo del cuidado al paciente 5 .

Según la Ley sobre "Modalidades Formativas Laborales" $\mathrm{N}^{\circ} 28518$, en el artículo 12 refiere: "Del aprendizaje con predominio en el Centro de Formación Profesional: Practicas preprofesionales, es la modalidad que permite a la persona en formación durante su condición de estudiante aplicar sus conocimientos, habilidades $y$ aptitudes mediante el desempeño en una situación real de trabajo"6. Fernández refirió que esta ley es una forma de dar oportunidad a los estudiantes durante su etapa de preprofesional, ya que el estudiante de enfermería va a aprender durante las experiencias clínicas que se presenten en el ámbito hospitalario, la cual es uno de los principales factores para la adquisición tanto de conocimientos como también de habilidades, aprendiendo a brindar un cuidado optimo y satisfactorio en cuanto a las necesidades que presente el paciente?.
Estudios realizados con un grupo de internos de enfermería demostraron que $73 \%$ tuvo problemas con los procedimientos que realiza para el cuidado al paciente ${ }^{8}, 56 \%$ tuvo dificultades para la organización, funcionamiento y dinámica de la unidad $^{9}, 32 \%$ con el proceso de cuidados, $26 \%$ en la comunicación con el paciente/familia/equipo, $23 \%$ en la responsabilidad profesional ${ }^{10}, 11,2 \%$ sensaciones de temor para preguntar y vergüenza de equivocarse $y$ recibir mal trato de las enfermeras 5 , por lo que los hace susceptible a cometer errores para el cuidado ${ }^{11}$. Sin embargo, al final de sus prácticas preprofesionales, $76,8 \%$ de los internos refirieron que las prácticas favorecen su adquisición de habilidades ${ }^{12}$.

La realidad evidencia que el desarrollo de habilidades para un cuidado holístico ${ }^{13}$, no es fácil para el interno, quien tiene que integrar saberes como: Saber conocer, saber ser, saber hacer y saber convivir14. Según los mismos internos durante el internado va disminuyendo la frustración, mejorando sus habilidades para el cuidado de la persona, pero esto es variable, así se señala que $41 \%$ de los estudiantes tuvo un buen desempeño en cuanto a sus habilidades, mientras que $31 \%$ un desempeño excepcional ${ }^{14}$ y $28 \%$ requiere de un mejoramiento en sus habilidades enfermeros para el cuidado a los pacientes ${ }^{15}$.

La realidad problemática descrita, basada en la evidencia, es la misma que se observa a nivel de la región Lambayeque, graficada en la existencia de seis escuelas de enfermería y un aproximado de 132 postulantes a las sedes de internado ofrecidas por el Ministerio de Salud (MINSA) por cada semestre, lo que urge profundizar para determinar los aspectos que caracterizan los programas de internado hospitalario y el proceso de adquisición de habilidades ${ }^{16}$.

Frente a la situación descrita anteriormente, el objetivo general fue determinar el nivel de adquisición de habilidades para el cuidado en internos hospitalarios de enfermería, Lambayeque 2020. Como objetivos específicos se plantearon: determinar el nivel de adquisición de habilidades 
en la dimensión Principiante en internos hospitalarios de enfermería, Lambayeque 2020 y determinar el nivel de adquisición de habilidades en la dimensión Principiante Avanzado en internos hospitalarios de enfermería, Lambayeque 2020.

\section{Metodología}

El estudio fue abordado mediante un enfoque cuantitativo descriptivo ${ }^{17}$, donde se especifica los atributos con que los internos de enfermería caracterizan la adquisición de habilidades en la práctica clínica, con la aplicación de un instrumento estandarizado tipo escala de Likert y procesado estadísticamente ${ }^{18}$.

Es una investigación descriptiva simple ${ }^{19}$, donde se buscó recopilar información cuantificable para ser utilizada en el análisis estadístico de la muestra de población, permitiendo especificar la naturaleza de cada una de las dimensiones de la adquisición de habilidades.

La población estuvo constituida por 132 internos hospitalarios de enfermería de la región de Lambayeque 20 . La muestra fue censal, puesto que se seleccionó el $100 \%$ de la población, por considerarse representativa y manejable, lográndose abordar todas las unidades de investigación ${ }^{21}$. La muestra fue seleccionada considerando además estos criterios: 1) Internos de enfermería que se encuentren realizando sus prácticas preprofesionales en la mitad del programa del internado. 2) Internos de enfermería que acepten participar en la investigación.

Para la recolección de datos se utilizó un instrumento estandarizado tipo escala de Likert para evaluar el nivel de adquisición de habilidades para el cuidado en internos hospitalarios de enfermería, el cual ha sido rediseñado y operacionalizado en coherencia con el marco conceptual y en la perspectiva de Patricia Benner.
El instrumento estuvo estructurado en dos dimensiones, con un total de veintiún ítems, que evaluaron la adquisición de habilidades en internos de enfermería de pregrado. El instrumento fue validado por juicio de expertos, considerando que su experticia fuera temática y metodológica, considerándose un total de tres jueces.

Así mismo, se procedió a realizar el análisis de consistencia interna y externa, mediante: a) Consistencia interna, un análisis juicio de expertos mediante la prueba binomial, llegándose a la conclusión la proporción de preguntas aceptadas por el juez $\mathrm{N}^{\circ} 1,2,3$ para el instrumento fue de $85 \%$, lo cual es un valor aceptable y aplicando prueba piloto con 100 internos 2019-II en la fecha de 25/11/19 hasta el 22/12/19, utilizando el programa estadístico SPSS 25 se obtuvo un alfa de Cronbach de 0.7 , lo cual indica que el instrumento tiene buena $y$ adecuada consistencia interna 22 y; b) Consistencia externa, obtenida mediante revisión del comité metodológico de la escuela de enfermería, y revisión del comité de Ética de la Facultad de Medicina.

La investigación una vez que fue aprobada con la resolución N 650-2019-USAT-FMED. A partir del $25 / 02 / 20$ se procedió aplicar el instrumento a los 132 internos hospitalarios de enfermería de la región de Lambayeque, con plaza adjudicada del semestre 2020-I mediante los datos publicados por la Geresa-Lambayeque 20 . Debido a las medidas adoptadas por la pandemia COVID-19 con Decreto Supremo que declara el estado de emergencia Nacional $\mathrm{N}^{\circ} 044-2020-\mathrm{PCM}^{23}$ y la restricción de la práctica de internado, solo a la mitad de la muestra se le aplicó de forma presencial, mientras que la otra mitad se ejecutó de forma virtual. Se utilizó el programa informático Microsoft Word para luego enviarlo por la aplicación WhatsApp de cada sujeto de estudio, explicándoles el objetivo de la investigación mediante la hoja informativa, además de cómo estuvo constituido el 
instrumento, se dejó en claro que la encuesta es de forma anónima. Se estimó un tiempo promedio de 10 minutos para responder las preguntas estipuladas en la escala de Likert.

Después de la ejecución de los instrumentos, se procesó los datos mediante el programa estadístico SPSS versión 25. Para ello, se tuvo en cuenta lo siguiente:

1) Las primeras 19 preguntas estuvieron constituidas por 5 alternativas de valor para todo el instrumento: TD: Totalmente en desacuerdo (1 pto), ED: En desacuerdo (2ptos), NIA NID: Ni de acuerdo ni en desacuerdo (3pts), DA: De acuerdo (4pts), TA: Totalmente de acuerdo (5pts) por dimensión, 2) Las dos últimas preguntas constituida por 5 alternativas que evalúan en cuantas semanas adquirieron las habilidades: en la primera dimensión ( 1 semana, 2 semanas, 3 semanas, 4 semanas) y en la segunda dimensión (5 semanas, 6 semanas, 7 semanas, 8 semanas), 3) A los resultados de cada dimensión se le dio un valor final de la siguiente manera: a) En la primera dimensión: Muy buena (37-45), Buena (28-36), Regular (19-27), Deficiente (9-18). b) En la segunda dimensión: Muy buena (41-50), Buena (31-40), Regular (21-30), Deficiente (10-20), 4) a la suma de ambas dimensiones se le dio un valor de: Muy buena (78-95), Buena (59-77), Regular (40-58), Deficiente (19-39), 5) Para explorar los datos mediante el análisis descriptivo, se tuvo en cuenta los objetivos planteados. Por último, se elaboró los resultados mediante cuadros y para luego realizar la discusión, las debidas conclusiones y recomendaciones.

En cuanto a los criterios éticos de la presente investigación, se tomaron en cuenta los siguientes: Se basó en el criterio de autonomía, brindándoles la hoja informativa para que posteriormente por respeto al sujeto de estudio tengan la oportunidad de decidir si quieren o no quieren participar en la investigación, por otro lado, en el criterio de autenticidad, considerando al Software anti-plagio Turnitin, en la cual se obtuvo un $24 \%$ de similitud. Además, se consideró el criterio de principio de no maleficencia, pues se dio de forma anónima, de tal forma que no se expongan a situaciones que les ocasionen daños, ya sean graves o permanentes. Finalmente, se consideró como criterio ético el principio de justicia, pues la investigadora aplicó el instrumento brindando un trato justo y pertinente a los sujetos de estudio durante la ejecución de la investigación ${ }^{24}$.

\section{Resultados}

Para una mejor explicación, los resultados del estudio sobre el nivel de adquisición de habilidades para el cuidado en internos hospitalarios de enfermería en el año 2020-I, se realizó un análisis global y por dimensiones que dieron respuesta a los objetivos planteados.

Medición Global de la Adquisición de Habilidades. Del $100 \%$ de internos de enfermería, el $75,8 \%$ aseveró que su adquisición de habilidades fue muy buena durante el internado hospitalario, mientras que solo el $0,8 \%$ piensa que no.

Tabla $\mathrm{N}^{\circ} \mathrm{l}$ :

Nivel de adquisición de habilidades para el cuidado en internos hospitalarios de enfermeria. Lambayeque, 2020

Adquisición de Habilidades Total

\begin{tabular}{lcc}
\hline Nivel & $\mathrm{N}^{\circ}$ de encuestados & Porcentaje \\
\hline Muy Buena & 100 & $75,8 \%$ \\
Buena & 30 & $22,7 \%$ \\
Regular & 1 & $0,8 \%$ \\
Deficiente & 1 & $0,8 \%$ \\
\hline Total & 132 & $100 \%$
\end{tabular}

Fuente: Encuesta de adquisición de habilidades 
Adquisición de Habilidades según dimensiones:

Tabla $N^{\circ}$ 2:

Nivel de adquisición de habilidades en la dimensión Principiante en internos hospitalarios de enfermería. Lambayeque, 2020

Dimensión Principiante

Nivel $\mathrm{N}^{\circ}$ de encuestados Porcentaje

\begin{tabular}{lcc}
\hline Muy Buena & 85 & $64,4 \%$ \\
Buena & 45 & $34,1 \%$ \\
Regular & 1 & $0,8 \%$ \\
Deficiente & 1 & $0,8 \%$ \\
\hline Total & 132 & $100 \%$
\end{tabular}

Fuente: Encuesta de adquisición de habilidades.

El $64,4 \%$ de los internos de enfermería afirmaron que el nivel de adquisición de habilidades según la dimensión principiante es muy bueno y el $0,8 \%$ consideró que fue deficiente.

Tabla $\mathrm{N}^{\circ}$ 3:

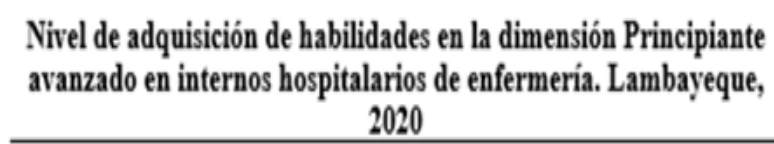

\begin{tabular}{lcc}
\multicolumn{3}{c}{ Dimensión Principiante Avanzado } \\
\hline Nivel & $\mathrm{N}^{\circ}$ de encuestados & Porcentaje \\
\hline Muy Buena & 108 & $81,8 \%$ \\
Buena & 22 & $16,7 \%$ \\
Regular & 1 & $0,8 \%$ \\
Deficiente & 1 & $0,8 \%$ \\
\hline Total & 132 & $100 \%$
\end{tabular}

Fuente: Encuesta de adquisición de habilidades.

El $81,8 \%$ de los internos de enfermería alegaron que el nivel de adquisición de habilidades según la dimensión principiante avanzado es muy buena y el $0,8 \%$ consideró que fue deficiente.

\section{Discusión}

A partir de los resultados encontrados, de forma global se obtuvo que la adquisición de habilidades del $75,8 \%$ de internos hospitalarios de enfermería fue muy buena, el $22,7 \%$ buena, mientras que solo el $0,8 \%$ piensa que no. Estos resultados se deben a que existe una deficiencia de enfermeras en el Perú, conllevando a que los internos de enfermería al estar en el campo clínico formen parte del recurso humano que colabora en el cuidado al paciente, por lo que esto les favoreció en su adquisición de habilidades.

Coincidiendo con Valencia, Bascur y Cáceres ${ }^{25}$, refieren que el $9 \%$ de sus estudiantes de enfermería

presentan una muy buena habilidad del cuidado durante sus prácticas preprofesionales. Este resultado es de esperarse, ya que a pesar de que las escuelas de enfermería de todo el mundo tienen la misma misión de formar profesionales con calidad y humanismo, pero la realidad cambia en cada país, de tal forma que las practicas son simuladas en los primeros años de estudio, posteriormente les surgen limitantes evidenciándose en la falta de iniciativa y autoconfianza en sus conocimientos aplicándolos en el paciente.

Por otro lado, un $55 \%$ de los estudiantes se sitúa en un nivel regular, $y$ esto es debido a que la adquisición de habilidades no es fácil, al intentar aprender diferentes procedimientos, primando el temor e inseguridad. El interno debe demostrar que es capaz de aplicar los conocimientos teóricos a la práctica clínica ${ }^{3}$. De forma que no todos los internos de enfermería logran adquirir las competencias necesarias, ya que de la noche a la mañana no se adquieren rápidamente las habilidades, pero al menos en el campo clínico se ofrece la oportunidad para la obtención de estas y para la resolución de situaciones clínicas ${ }^{26}$.

A diferencia de Concha y López ${ }^{27}$, refieren que el desempeño de las enfermeras recién egresadas 
aun es deficiente, puesto que no se observan habilidades en cuanto a la toma de decisiones, falta de iniciativa, planificación y resolución de conflictos; y todo esto es porque aún son principiantes en el campo clínico. Lo mismo refiere Benitez $^{8}$, que el $90 \%$ manifestó tener iniciativa para realizar diversos procedimientos en la práctica hospitalaria, mientras que el $10 \%$ dijo no tener iniciativa. Es por ello por lo que se afirma que el realizar alguna actividad o procedimiento por uno mismo; se debe a la seguridad en el conocimiento enfermero que cada uno posee, para ponerlo en práctica lo aprendido; más aún cuando se va a iniciar un proceso de práctica.

De igual forma, Torres ${ }^{14}$, presenta resultados opuestos, debido a que, según la percepción de las enfermeras, $60 \%$ de los internos de enfermería muestran desfavorable adquisición de habilidades, debido a sus inseguridades $y$ temores en los procedimientos, en el diagnóstico, planificación y la toma de decisiones en la resolución de problemas para un mejor cuidado al paciente.

Con relación a la dimensión principiante, se muestra que $64,4 \%$ de los internos de enfermería afirmaron que el nivel de adquisición de habilidades es muy bueno, $34,1 \%$ bueno y $0,8 \%$ consideró que fue deficiente. La razón de estos resultados es que gran parte de los internos al inicio de las practicas se enfrentan a una nueva situación con inseguridad en el conocimiento y la práctica, además que desempeñan por primera vez el cuidado en una nueva área clínica, se rigen de reglas, normas o indicaciones para brindar cuidados al paciente, vinculando los conocimientos teóricos con lo practico en situaciones de baja complejidad y luego formular un plan de cuidados básicos de enfermería, como consecuencia de todo lo vivido desarrollan competencias después de considerables situaciones.

Al respecto Florian', en el cual su estudio fue para enfermeras, en la dimensión Principiante en un servicio de emergencia; $58 \%$ valoró que desarrolló habilidades en un nivel bueno, $36 \%$ regular; y solo $6 \%$ de las enfermeras lograron desplegar una adquisición de habilidades muy buena en su práctica clínica. Este resultado obtenido se debe a que la enfermera por primera vez se enfrenta a una nueva situación, no siendo experta para desempeñarse en una nueva área que lo considera desconocida.

Guía ${ }^{28}$, con respecto a la seguridad del conocimiento del estudiante, los conocimientos teóricos son considerados herramientas muy importantes porque brindan los conceptos pertinentes para la ejecución del cuidado y tener buen desenvolvimiento en el inicio de sus prácticas pre profesionales, las habilidades en cambio, se van obteniendo en el quehacer diario, evidenciándose en su estudio que $40 \%$ opina que tienen seguridad de los conocimientos que poseen para emplearlo en la práctica, mientras que $60 \%$ opina lo contrario.

Benite $z^{8}$, difiere en su investigación, ya que obtuvo que $95 \%$ de los estudiantes de enfermería poseen los conocimientos necesarios para iniciar la práctica hospitalaria; considerándose, como fortaleza para los estudiantes porque pueden demostrar su capacidad de comprensión e interacción en el entorno hospitalario, así como, para conocer los métodos de trabajo, el funcionamiento de la unidad y el rol de la enfermera para adaptarse a la dinámica de trabajo en el área de prácticas.

Por lo tanto, en la dimensión principiante, del $100 \%$ de internos de enfermería se muestra que $47 \%$ logró su adquisición de habilidades en 2 semanas, $28 \%$ en 4 semanas y $11,4 \%$ en 3 semanas. Este resultado coincide con lo que refiere Pérez y García ${ }^{29}$, que afirman, que los internos de enfermería desde un inicio de la práctica participan de forma activa las situaciones que se presentan en el ámbito hospitalario, exigiéndose de tomar sus propias iniciativas $y$ toma de decisiones para la obtención de habilidades. Como resultado los estudiantes de enfermería progresivamente fueron 
comprendiendo al aplicar el conocimiento teórico a la práctica, mediante análisis para clasificar, relacionar y evaluar en base a estándares, criterios específicos y sobre todo con los diagnósticos enfermeros ${ }^{30}$.

En relación con la dimensión principiante avanzado, se demuestra que $81,8 \%$ de los internos de enfermería afirmaron que el nivel de adquisición de habilidades es muy bueno, 16,7\% es bueno y $0,8 \%$ consideró que fue deficiente, este resultado es similar al obtenido en otro estudio realizado con enfermeras, donde $64 \%$ ejecutaron una buena adquisición de habilidades en la práctica y $36 \%$ refieren que muy buena ${ }^{1}$. La razón de estos resultados es que los internos ya utilizan el pensamiento crítico basado en estándares institucionales, mostrando una mayor capacidad para ejecutar planes de atención de cuidado enfermero, evidenciándose en la valoración, diagnóstico, planificación, ejecución y evaluación al paciente, además de mostrar experiencia suficiente para detectar distintos aspectos de una situación y sobre todo una mayor capacidad para evitar riesgos según el estado de salud en la que se encuentra el paciente.

Benite $z^{8}$, afirma que es fundamental considerar que el cuidado es lo que sustenta a Enfermería como profesión y que debe ser brindado con calidad y calidez. Los resultados mostraron que más del $73 \%$ de los estudiantes tuvieron una buena adquisición de habilidades evidenciándose en un mejor desenvolvimiento, gracias al apoyo de las enfermeras de turno.

A diferencia, Cervantes ${ }^{4}$, en su investigación de las competencias de internos de enfermería bajo la perspectiva del profesional de enfermería, se obtuvo que $39.1 \%$ con un nivel regular, estos resultados son de suponerse ya que debido a la falta de experticia los estudiantes aun no asumen las responsabilidades, además que aun manifiestan que necesita ayuda para resolver problemas en el cuidado según necesidades del paciente.
Araya, Bianchetti, Torres, Veliz ${ }^{9}$, refiere que las experiencias que obtendrán los estudiantes de enfermería serán de diversas situaciones clínicas que se presentan en los hospitales, es por eso por lo que la estadía debe ser suficiente para conseguir la adquisición de habilidades como principiante avanzado que se desea lograr en la práctica. Es así como se evidencia en esta investigación que del $100 \%$ de internos de enfermería, 45,5\% logró su adquisición de habilidades en 8 semanas y 7,6\% en 7 semanas.

Castillo, Llaurado, Aliberch y Rodriguez ${ }^{31}$, refieren que, al evaluar las habilidades de sus estudiantes de medicina y enfermería en un curso de 6 meses de constante práctica, se obtuvo un buen resultado en la obtención de habilidades y este será más duradero en la medida que su estancia en el centro hospitalario también lo sea. Por el contrario, Benite $z^{8}$, refiere que, en cuanto al tiempo dedicado para la práctica hospitalaria, $67 \%$ consideró que no era suficiente la estadía en las diferentes áreas del campo clínico y 33\% consideró que el tiempo era perfecto para adquirir la experticia como enfermera general. Estos resultados se deben al incremento del alumnado en las escuelas de enfermería, provocando una limitación en la adquisición de habilidades de los estudiantes.

El aprendizaje basado en problemas y las situaciones reales criticas dan un buen resultado para la adquisición de habilidades de comunicación, habilidades de organización y de gestión personal, actitudes y valores del desarrollo profesional como la autonomía, trabajo en equipo, compromiso personal mostrando iniciativa de brindar el cuidado y responsabilidad que conlleva atender a un paciente ${ }^{32}$, de tal forma que aprendieron a solucionar creativamente los problemas, adaptándose con flexibilidad a las nuevas situaciones, propias de una sociedad en continuo cambio ${ }^{33}$.

Finalmente, las practicas preprofesionales de enfermería, es una etapa de cambio para el estudiante de enfermería, pasando de ser 
observador imparcial a convertirse en un ejecutor comprometido en realizar la acción de cuidado ${ }^{34}$, ayudando a las internas de enfermería a integrarse como parte del equipo de trabajo de una Institución hospitalaria, laborando en colaboración, no solo con las enfermeras, sino también con el resto del equipo multidisciplinario adaptándose progresivamente a un ámbito totalmente diferente donde las tareas interpersonales y colaborativas requieren del destaque de sus competencias 5 ; así mismo, ayudó a disminuir sus inseguridades para enfocarse en las nuevas habilidades que adquirieron $y$ paulatinamente lograron apropiarse de las intervenciones de cuidado de enfermería, logrando realizarlas de forma satisfactoria ${ }^{35}$. La visión fue cambiando a medida que el estudiante experimenta vivencias de cuidado, integrando todos los conocimientos aprendidos, evaluando diferentes aspectos críticos, biológicos, psicológicos, según la condición de salud del paciente, de esta forma adquirió un buen nivel de formación ${ }^{36}$.

\section{Conclusiones}

La adquisición de habilidades se considera como la obtención de un dominio. En enfermería está diseñado por niveles de competitividad y dificultad de aprendizaje, los cuales serán logrados progresivamente al aplicar los distintos conocimientos y técnicas adquiridos durante la formación de enfermería y recurrente atención al paciente. Para los internos de enfermería significa una lucha constante, afrontando sus propios miedos e inseguridades, al enfrentarse a nuevas situaciones demostrando iniciativa, apoyándose de soluciones reflexivas premeditadas aprendiendo a detectar diferentes problemas de salud (intuición), todos estos logros son fruto de varias horas de observación directa del paciente.

Existen limitantes para alcanzar un nivel de adquisición de habilidades óptimas por parte de los estudiantes, debido al incremento de la oferta educativa en las escuelas de enfermería en el país, lo que no es correlativo con la oferta de campos clínicos, lo que conlleva a que los internos de enfermería tengan que compartir un mismo servicio con otro compañero, causando limitada asignación de casos y por ende merma en la adquisición de habilidades.

De manera global, el nivel de adquisición de habilidades para el cuidado en internos hospitalarios de enfermería. fue muy bueno $(75,8 \%)$, los estudiantes reconocieron que sus prácticas preprofesionales (internado) fue una buena oportunidad para alcanzar las competencias, asumiendo que el principal factor es la práctica constante, el número de casos asignados y la rotación por diferentes servicios, permitiéndoles brindar un cuidado eficiente $y$ satisfactorio del paciente y sus necesidades.

De manera específica la adquisición de habilidades en el nivel principiante es muy buena $(64,4 \%)$, pese a que $47 \%$ de los internos hospitalarios de enfermería manifestaron que en las dos primeras semanas se enfrentaron a diferentes situaciones clínicas experimentando una práctica incipiente e insegura, la cual les sirvió para tomarlo como experiencia específica, relacionando el conocimiento teórico-practica, ayudándoles así a adquirir sus habilidades como principiante.

En la dimensión Principiante Avanzado el nivel de adquisición de habilidades fue percibido como muy buena $(81,8 \%)$, de las cuales $45,5 \%$ manifestó haber logrado su adquisición de habilidades en ocho semanas. Reconocieron que, a este tiempo, desarrollaron un pensamiento crítico, mayor capacidad de intuición, actuaron basados en normas y protocolos de la institución, una mayor capacidad de realizar una valoración, diagnóstico, planificación, ejecución y evaluación, además de mayor facilidad para detectar riesgos que puedan complicar el estado de salud del paciente, asegurando que la experiencia fue suficiente para detectar los distintos aspectos de una situación clínica. 


\section{Bibliografía}

1. Florian F. Práctica de la Enfermera según la Teoría Principiante a Experta del servicio de emergencia del Hospital Regional Docentes las Mercedes [Tesis licenciatura en internet]. Pimentel: Universidad Señor de Sipán; 2018 [consultada 30 May 2019]. 74p. Disponible en: https://cutt.ly/8jwN7wH

2. Burgos M, Paravic T. Enfermería como profesión. Revista Cubana de Enfermería [internet]. 2009 [consultada 30 May 2019]; 25(2): 1-9p. Disponible en: https://cutt.ly/CjwMpoa

3. Jiménez J, More M. Competencias de los internos de enfermería de la Universidad Nacional de Tumbes en las prácticas pre profesionales Hospital Regional Jamo II-2 [Tesis licenciatura en internet]. Tumbes: Universidad Nacional de Tumbes; 2016 [consultada 30 May 2019]. 58p. Disponible en: https://cutt.ly/zoE7s7d

4. Cervantes M. Percepción del profesional de enfermería de Essalud y Minsa sobre la competencia social del interno de enfermería de la Universidad Nacional Jorge Basadre Grohmann. [Tesis licenciatura en internet]. Tacna: Universidad Nacional Jorge Basadre Grohmann; 2015 [consultada 3 Agos 2019]. 116p. Disponible en: https://cutt.ly/EjwMviN

5. Herrera L, Tejada A. Vivencias de estudiantes de enfermería durante su internado hospitalario- Hospital Regional Docente Las Mercedes [Tesis licenciatura en internet]. Lambayeque: Universidad Nacional Pedro Ruiz Gallo; 2017 [consultada 30 May 2019]. 71 p. Disponible en: https://cutt.ly/JjwMSGg

6. Ley sobre modalidades formativas laborales. Ley 28518 . Art 12. Mayo 23 de 2005 (Perú).

7. Fernández D. Transición de la Teoría a la Práctica: Desde la perspectiva de los internos de enfermería de la Universidad Señor de Sipán, 2017 - Pimentel [Tesis licenciatura en internet]. Pimentel: Universidad Señor de Sipan; 2019 [consultada 01 Abr 2020]. 83p.
Disponible en: https://cutt.ly/kjwMBFz

8. Benitez M. Fortalezas y debilidades en el inicio de prácticas hospitalarias de los estudiantes de la carrera de enfermería de la Universidad Nacional de Loja. [Tesis licenciatura en internet]. Loja: Universidad Nacional de Loja; 2016. [consultada 30 May 2019]. 55p. Disponible en: https://cutt.ly/LjwM6Hq

9. Araya S, Bianchetti A, Torres J, Veliz L. Expectativas y experiencias de aprendizaje en la práctica profesional de estudiantes del área de la salud. Sociedad Cubana de Educadores en ciencias de la salud[internet]. 2018 [consultada 30 May 2019]; 32(1): $15 \mathrm{p}$. Disponible en:_https://cutt.ly/vjw1 uQ3

10. Carrasco T, Sanchez A. La transición de la teoría a la práctica: desde la percepción de los estudiantes del vii ciclo [Tesis licenciatura en internet]. Pimentel: Universidad Señor de Sipan; 2016 [consultada 30 May 2019]. 83p. Disponible en: https://cutt.ly/6jw1 IW1

11. Paredes M. Competencias cognitivas del profesional de Enfermería en situaciones de emergencia en el Hospital Sergio Bernales Collique, Comas 2017. [Tesis licenciatura en internet]. Lima: Universidad Cesar Vallejo; 2017 [consultada 3 Agos 2019]. 219p. Disponible en: https://cutt.ly/loE5TTm

12. Rojas J, Rivera L, Morera M. Aspectos pedagógicos en Educación enfermería: revisión integradora. Investigación y Educación en Enfermería [Internet]. Set 2018 [consultada 01 Abr 2020]; 36 (3): 1-17. Disponible en: https://cutt.ly/yjw1Yv3

13. Barrientos L, Escalante E. Satisfacción del interno(a) con la carrera de enfermería y su relación con. el desempeño según el profesional de enfermería del Hospital Regional de Ayacucho-2014 [Tesis licenciatura en internet]. Ayacucho: Universidad Nacional de san Cristóbal de Huamanga; 2015 [consultada 12 Agos 2019]. 98p. Disponible en: https://cutt.ly/hjwl G0E

14. Torres W. Percepción de las enfermeras sobre el cuidado que brindan los internos de enfermería Instituto Nacional de Ciencias 
Neurológicas Barrios Altos mayo 2017 [Tesis licenciatura en internet]. Lima: Universidad Privada San Juan Bautista; 2018 [consultada 3 Agos 2019]. 76p. Disponible en: https://cutt.ly/djw1 ZI3

15. Cusihuallpa C. Calidad de las prácticas preprofesionales según percepción de los internos de enfermería. Hospital Militar Central. [Tesis licenciatura en internet]. Lima: Universidad Cesar Vallejo; 2017 [consultada 17 Jun 2019]. 106p. Disponible en: https://cutt.ly/Ejw110u

16. Gonzales C. Competencias que desarrolla una enfermera en etapa principiante avanzada [internet]. 2017 [consultada 30 Mayo 2019]; 28(1): 13p. Disponible en: https://cutt.ly/0jw13YE

17. Ñaupas $H$, Valdivia $M$, Palacios J, Romero $H$. Metodología de la investigación cuantitativacualitativa y redacción de la tesis. 5 ta edición. Bogotá: Ediciones de la U; 2018. 559pg.

18. Ortiz F, García M. Metodología de la investigación: el proceso y sus técnicas. México: Limusa; 2016. 179pg.

19. Muñoz C. Como elaborar y asesorar una investigación de tesis. 3era edición. México: Pearson Educación; 2015. 488pg.

20. Gestión y desarrollo del potencial humano. Área de capacitación [Internet]. Lambayeque: Gerencia Regional de Salud (Perú); 2020 [consultada 04 May 2020]. Disponible en: https://cutt.ly/ljw0rT5

21. Hernández R. Fernández C. Baptistas P. Metodología de la investigación. 6th ed. México D.F.: Mc Graw Hill; 2015. 1-613 p.

22. Molina, JA Bojórquez, et al. "Utilización del alfa de Cronbach para validar la confiabilidad de un instrumento de medición de satisfacción del estudiante en el uso del software Minitab MISP." 11th LACCEI Latin American and Caribbean Conference for Engineering and Technology (LACCEI'2013) "Innovation in Engineering, Technology and Education for Competitiveness and Prosperity" August. 2013.

23. Decreto Supremo que declara Estado de
Emergencia Nacional por las graves circunstancias que afectan la vida de la Nación a consecuencia del brote del COVID-19 [Internet]. Perú: Nº44-2020-PCM, 2020 [consultada 4 May 2020]. Disponible en: https://cutt.ly/kjw0aXF

24. Acevedo I. Aspectos éticos en la investigación científica. Ciencia y enfermería[internet]. 2002 [consultada 24 Oct 2020]; 8(1): 15-18. Disponible en: https://cutt.ly/Qjw0gez

25. Valencia C, Bascur J, Cáceres A. Habilidad de cuidado en estudiantes de enfermería de una Universidad privada del sur de Chile. BENESSERE - Revista de Enfermería. [internet]. 2019 [consultada 13 Jun 2020]; 4(1): 43-52. Disponible en: https://cutt.ly/Wjw0R3p

26. Tafur D. Experiencias del estudiante de enfermería respecto al internado hospitalario, Universidad Señor de Sipán 2019. [Tesis licenciatura en internet]. Perú: Universidad Señor de Sipán; 2019. [consultada 24 Oct 2020]. 67p. Disponible en: https://cutt.ly/ljw0SOz

27. Concha P, López I. Percepción de las enfermeras asistenciales sobre competencias actitudinales observadas en enfermeros recién egresados, Chile. Rev Educ Cienc Salud [internet]. 2015 [consultada 24 Oct 2020]; 12(1): 13-18. Disponible en: https://cutt.ly/ljw0Hfy

28. Guía M. Teoría y práctica en el desarrollo de las competencias de enfermería en pediatría. Revista de investigación en salud. [internet].2019 [consultada 24 de Octubre 2020]; 2(5): 84-91. Disponible en: https://cutt.ly/HjwOVti

29. Pérez M, García C. Valoración de los estudiantes de grado de enfermería sobre las competencias a adquirir durante su formación. Nure Inv. [internet]. Junio 2015 [consultada 24 Oct 2020]; 12(78): 1-16. Disponible en: https://cutt.ly/Kjw00Wl 
30. Arcos M. rueda D. Balseca J. Análisis de la importancia del desarrollo del pensamiento crítico en la formación de estudiantes de enfermería. Revista Med [internet]. Diciembre 2017 [consultada 24 Oct 2020] 4(1): 947-962. Disponible en: https://cutt.ly/Tjw9uz5

31. Castillo J. Llaurado M. Aliberch A. Rodriguez E. Comparación en la adquisición y retención de competencias en soporte vital entre formación uni 0 interdisciplinar. Estudio cuasiexperimental. Atención primaria [internet]. Mayo 2020 [consultada 24 Oct 2020]. 52(5): 367-368. Disponible en: https://cutt.ly/Yjw3fYC

32. Moran J. Ruiz P. Ruiz P. Calvo R. Metodologías complementarias de aprendizaje para la adquisición de competencias en la formación de especialistas y actividades profesionales confiables. Educación médica [internet]. 2020 [consultada 24 Oct 2020]. 21(5): 328-337. Disponible en: https://cutt.ly/rjw3Q19

33. Moya C. Pulgar C. Trajtmann A. Grupos de encuentro: desarrollo de habilidades para la humanización del cuidado, a partir de la experiencia de aprendizaje de estudiantes de enfermería. Foro educacional [internet]. Octubre 2019 [consultada 24 Oct 2020]; 33(1): 53-77. Disponible en: https://cutt.ly/jijw3AbF

34. Lara C. Modelo Innovador: Educación para la Integración y Desarrollo de Habilidades de Cuidado de los Estudiantes de Enfermería [Tesis licenciatura en internet]. México: Universidad Autónoma de Chihuahua; 2013 [consultada 13 Jun 2020]. 76 p. Disponible en: https://cutt.ly/Cjw8ao7

35. Naranjo J, Báez O, Delgado A, Álvarez K, Martínez N. Formación de habilidades profesionales en los estudiantes. Revista de Ciencias Médicas de Pinar del Río [internet]. 2018 [consultada 13 Jun 2020]; 22(3): 523$533 . \quad$ Disponible en: http://scielo.sld.cu/pdf/rpr/v22n3/rpr13318 .pdf

36. Guzmán V. Valencia. Vivencias de los estudiantes de enfermería respecto al cuidado brindado durante sus prácticas formativas [Tesis licenciatura en internet]. Argentina: Universidad de Córdoba; 2019 [consultada 24 Oct 2020]. 57 p. Disponible en: https://cutt.ly/4jw8RqZ 